\section{From qualitative radiological cues to machine learning: MRI-based diagnosis in neurodegeneration}

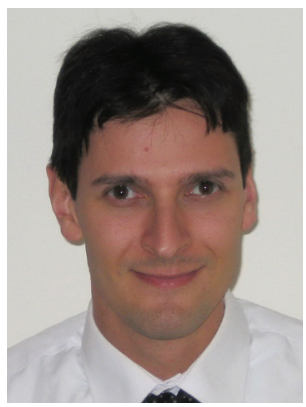

Peter Bede*
"With continued advances in quantitative imaging, the gap between research MRI protocols and clinical radiology continues to widen.”

First draft submitted: 4 December 2016; Accepted for publication: 5 December 2016; Published online: 20 January 2017
Neurodegenerative conditions are generally regarded as a diverse group of neurological syndromes, with distinct pathological features, unique atrophy patterns, diseasespecific progression rates and genetic susceptibility. Nonetheless, they share a tures, such as a long presymptomatic phase, relentless progression, significant diagnostic challenges, lack of effective diseasemodifying therapies and late recruitment into clinical trials [1]. Diagnostic delay in neurodegenerative conditions often leads to unnecessary interventions, impedes early genetic screening and counseling, delays introduction of neuroprotective drugs and recruitment into clinical trials $[2,3]$. In amyotrophic lateral sclerosis (ALS), the average diagnostic delay from symptom onset to definite diagnosis is approximately 12 months, which not only delays recruitment to pharmaceutical trials, but misdiagnosis of ALS to other conditions may lead to unnecessary interventions [4]. Significant diagnostic delay also hampers the management of other neurodegenerative conditions such as primary lateral sclerosis, hereditary spastic number of strikingly common clinical fea- paraplegia, corticobasal degeneration, ataxia and dementia syndromes.

\section{Standard MRI in neurodegeneration}

Standard MRI in these conditions adds surprisingly little to the diagnosis and is primarily performed to rule out inflammatory or neoplastic etiologies. Standard clinical MRI pulse sequences are generally optimized for quick data acquisition, and often include large slice-gaps. Furthermore, standardized clinical protocols routinely incorporate pulse sequences which are not directly relevant to neurodegeneration. While most neurodegenerative conditions exhibit unique, disease-specific atrophy patterns, these are difficult to interpret qualitatively [5]. On routine clinical imaging, ALS is associated with precentral gyrus atrophy, behavioral variant frontotemporal degeneration exhibits orbitofrontal and dorsolateral prefrontal cortex atrophy, and Alzheimer's disease is associated with considerable temporoparietal and mesial temporal lobe atrophy. Huntington's disease is dominated by caudate head atrophy resulting in frontal horn enlargement, progressive supranuclear palsy is associated
*Author for correspondence: Quantitative Neuroimaging Group, Academic Unit of Neurology, Biomedical Sciences Institute, Trinity College Dublin, Ireland; Tel.: +353 1 8964497; Fax: +353 1 2604787; bedep@tcd.ie

\author{
KEYWORDS \\ - amyotrophic lateral sclerosis \\ - biomarker • classification \\ - machine learning \\ - neurodegeneration
}


“Despite the widespread use of machine-learning algorithms in information technology, banking, economics, meteorology and marketing, they have not been utilized to their full potential in clinical radiology." with relatively selective midbrain atrophy, and multiple system atrophy-C is associated with olivopontocerebellar degeneration on clinical MRI. These disease-specific atrophy patterns however are regarded as poorly specific, and are not sensitive enough to confirm the clinical diagnosis. Other qualitative imaging cues such as the 'hot-cross bun sign' of the pontocerebellar tracts in multiple system atrophy, 'wine glass appearance' of the corticospinal tracts in ALS, the 'mickey mouse appearance', 'hummingbird sign' or 'penguin sign' of the midbrain in progressive supranuclear palsy, the 'Pulvinar sign' and 'Hockey-stick sign' in Creutzfeldt-Jakob disease are occasionally observed but have no role in the diagnosis of the underlying condition. This is a missed opportunity as these signs reflect on the selective pathology of "disease-defining" key brain regions, which could be evaluated by quantitative imaging techniques and interpreted based on normative values. Single-plane measurement ratios and semi-quantitative scoring systems have also been proposed for neurodegenerative conditions such as the global cortical atrophy scale, magnetic resonance parkinsonism index, the medial temporal lobe atrophy score or the posterior atrophy score of parietal atrophy (PA/Koedam score), but these are seldom utilized in everyday clinical practice.

State-of-the-art imaging in the pipeline The limitations of subjective data interpretation in clinical radiology are in striking contrast with the advanced quantitative imaging protocols used in research. Diffusion tensor-imaging, highangular-resolution diffusion imaging and Q-ball vector analysis are just some of the white matter techniques which enable the reliable assessment of white matter integrity, characterization of crossing fiber anatomy and tractography [6]. Network and circuitry integrity can be objectively evaluated by novel diffusion techniques, connectomics and resting-state functional MRI (fMRI) protocols [7]. Atrophy patterns can be reliably mapped with density and cortical thickness analyses and compared with healthy controls [8]. Subcortical gray matter structures can be quantitatively characterized by volumetric, density and vertex analyses [9]. Biochemical and metabolic information can be ascertained from single voxels or from the entire brain using MR spectroscopy [10]. These quantitative neuroimaging methods have been extremely successful in capturing presymptomatic changes, phenotype-specific imaging signatures, genotype-specific patterns of degeneration and mapping progressive longitudinal changes in neurodegeneration [11]. Yet, despite their potential, these technologies have not been adapted for routine clinical use. With continued advances in quantitative imaging, the gap between research MRI protocols and clinical radiology continues to widen. While clinical reports continue to comment on 'volume loss', 'age-related changes', 'white matter hyperintensities' and 'global atrophy', high resolution research protocols can now accurately measure cranial nerve and spinal cord white matter integrity [12].

\section{Moving toward individual data interpretation}

Quantitative imaging studies have initially provided group-level observations and their value was primarily descriptive, characterizing disease- or phenotype-specific imaging traits. In recent years, however, there has been a marked shift from group-level analyses to individual data interpretation. With an ever-growing interest in diagnostic classification, binary logistic regression, random forest classification, naive Bayes algorithms and support vector machines have been increasingly applied to neurodegenerative conditions to classify blinded datasets as 'healthy' or 'pathological'. These methods remain experimental in radiology and have not filtered down to everyday clinical applications. This is somewhat surprising in an era, where classification techniques, pattern recognition models and machine learning are widely used in face recognition, speech recognition, consumer profiling, security screening and weather prediction. The statistical models behind these technologies are also extensively used for spam recognition, cybersecurity, traffic control, autonomous vehicles, economic projections, user preference prediction by online content providers, targeted advertising and fraud detection. Despite the widespread use of machine-learning algorithms in information technology, banking, economics, meteorology and marketing, they have not been utilized to their full potential in clinical radiology. In research, machinelearning methods have been explored in neurodegenerative conditions with varying degrees of success [6,13]. In ALS, smaller classification studies achieved relatively good diagnostic sensitivity based on diffusivity measures [14], but a large meta-analysis of several studies, suggests 
that the diagnostic value of corticospinal tract diffusion tensor-imaging metrics alone is limited [15]. A total of $71 \%$ diagnostic accuracy was achieved based on functional MRI metrics using a support-vector machine approach [16]. In ALS, we have achieved a diagnostic sensitivity of $85.7 \%$ and accuracy of $78.4 \%$ in an independent validation sample using binary logistic regression [17]. The advantage of binary logistic regression models is that instead of categorical classification labels, a quantitative diagnostic probability outcome is provided. Support vector machines or support vector networks are supervised learning models, nonprobabilistic binary linear classifiers $[16,18]$. The advantage of support vector learning is its regularization parameter, which helps preventing model overfitting. The advantage of random forest methods is that they are able to deal with unbalanced or missing data and runtimes are generally fast. However, random forests cannot predict beyond the range in the training data, and they may overfit datasets that are particularly noisy. The strength of naive Bayes approaches is that they tend to perform better compared with other models like logistic regression and less training data is generally required. In clinical applications where three-way classification is required, for example, 'specific condition', 'healthy control' or 'mimic neurodegenerative condition', naive Bayes is ideally suited to provide multiclass categorical classification. The disadvantages of naive Bayes methods include the assumption of independent predictors, poor probability estimations and requirement of smoothing techniques. One of the main shortcomings of existing classifier studies in neurodegeneration is the lack of validation in independent samples.

While the core pathology is different in various neurodegenerative conditions, the commonly used MRI pulse sequences and analysis pipelines are similar. With the recognition that neurodegenerative conditions have a long presymptomatic phase and MRI alterations can be captured in presymptomatic cohorts of pathological mutation carriers, evaluation of 'diseasedefining' brain regions are particularly promising in establishing an early diagnosis. The initiatives of setting up multicenter studies, validating cross-platform protocols and establishing multicenter data repositories are also analogous in various neurodegenerative conditions, for example, the Alzheimer's disease neuroimaging initiative, TRACK-HD or the Neuroimaging Society in ALS [19]. The other striking and under-reported feature of neurodegenerative conditions is the relative sparing of specific anatomical regions. The anatomical patterns of these 'unaffected' brain regions are just as condition-specific as the 'affected' regions. Unaffected regions have not been previously incorporated in diagnostic classification studies despite their obvious role in discriminating specific syndromes from other mimic neurodegenerative conditions [20].

\section{Conclusion}

MRI is a versatile and noninvasive diagnostic tool which is widely available in hospitals and research institutions. In the past few years, successful initiatives have been undertaken to classify blinded imaging data into 'healthy' or 'disease' groups and robust mathematical classification models have long been available. It is conceivable, that the diagnostic accuracy of classification models will increase considerably in neurodegeneration, thanks to ever-evolving MRI platforms and optimized machine-learning protocols which will integrate multiple imaging measures from multiple anatomical regions. We are likely to witness a radiological paradigm shift worldwide, transitioning from qualitative data interpretation to quantitative imaging, where normative references ranges will be increasingly utilized.

\section{References}

1 Schuster C, Elamin M, Hardiman O, Bede P. Presymptomatic and longitudinal neuroimaging in neurodegeneration - from snapshots to motion picture: a systematic review. J. Neurol. Neurosurg. Psychiatry 86(10), 1089-1096 (2015).

2 Cellura E, Spataro R, Taiello AC, La Bella V. Factors affecting the diagnostic delay in amyotrophic lateral sclerosis. Clin. Neurol. Neurosurg. 114(6), 550-554 (2012).
3 Zoccolella S, Beghi E, Palagano G et al. Predictors of delay in the diagnosis and clinical trial entry of amyotrophic lateral sclerosis patients: a population-based study. J. Neurol. Sci. 250(1-2), 45-49 (2006).

4 Wokke JH. Confounding effects of mimicking disorders in the early diagnosis of amyotrophic lateral sclerosis. Amyotroph. Lateral Scler. Other Motor Neuron Disord. 1(Suppl. 1), S61-S63 (2000).

5 Bede P, Hardiman O. Lessons of ALS imaging: pitfalls and future directions - a critical review. Neuroimage Clin. 4, 436-443 (2014).

6 Filippini N, Douaud G, Mackay CE, Knight S, Talbot K, Turner MR. Corpus callosum involvement is a consistent feature of amyotrophic lateral sclerosis. Neurology 75(18), 1645-1652 (2010).

7 Menke RA, Proudfoot M, Wuu J et al. Increased functional connectivity common to symptomatic amyotrophic lateral sclerosis and those at genetic risk. J. Neurol. Neurosurg. Psychiatry 87(6), 580-588 (2016). 


\section{EDITORIAL Bede}

8 Bede P, Bokde A, Elamin M et al. Grey matter correlates of clinical variables in amyotrophic lateral sclerosis (ALS): a neuroimaging study of ALS motor phenotype heterogeneity and cortical focality. J. Neurol. Neurosurg. Psychiatry. 84(7), 766-773 (2013).

9 Bede P, Elamin M, Byrne S et al. Basal ganglia involvement in amyotrophic lateral sclerosis. Neurology 81(24), 2107-2115 (2013).

10 Kalra S, Hanstock CC, Martin WRW, Allen PS, Johnston WS. Detection of cerebral degeneration in amyotrophic lateral sclerosis using high-field magnetic resonance spectroscopy. Arch. Neurol. 63(8), 1144-1148 (2006).

11 Bede P, Bokde ALW, Byrne $S$ et al. Multiparametric MRI study of ALS stratified for the C9orf72 genotype. Neurology 81(4), 361-369 (2013).

12 Bede P, Bokde AL, Byrne S, Elamin M, Fagan AJ, Hardiman O. Spinal cord markers in ALS: diagnostic and biomarker considerations. Amyotroph. Lateral Scler. 13(5), 407-415 (2012).

13 Wang S, Summers RM. Machine learning and radiology. Med. Image Anal. 16(5), 933-951 (2012).

14 Ben Bashat D, Artzi M, Tarrasch R, Nefussy B, Drory VE, Aizenstein O. A potential tool for the diagnosis of ALS based on diffusion tensor imaging. Amyotroph. Lateral Scler. 12(6), 398-405 (2011).

15 Foerster BR, Dwamena BA, Petrou M et al. Diagnostic accuracy of diffusion tensor imaging in amyotrophic lateral sclerosis: a systematic review and individual patient data meta-analysis. Acad. Radiol. 20 (9), 1099-1106 (2013).

16 Welsh RC, Jelsone-Swain LM, Foerster BR. The utility of independent component analysis and machine learning in the identification of the amyotrophic lateral sclerosis diseased brain. Front. Hum. Neurosci. 7,251 (2013)
17 Schuster C, Hardiman O, Bede P. Development of an automated MRI-based diagnostic protocol for amyotrophic lateral sclerosis using disease-specific pathognomonic features: a quantitative disease-state classification study. PLoS ONE 11(12), e0167331 (2016)

18 Orru G, Pettersson-Yeo W, Marquand AF, Sartori G, Mechelli A. Using support vector machine to identify imaging biomarkers of neurological and psychiatric disease: a critical review. Neurosci. Biobehav. Rev. 36(4), 1140-1152 (2012).

19 Muller HP, Turner MR, Grosskreutz J et al. A large-scale multicentre cerebral diffusion tensor imaging study in amyotrophic lateral sclerosis. J. Neurol. Neurosurg. Psychiatry 87(6), 570-579 (2016).

20 Bede P, Iyer PM, Schuster C et al. The selective anatomical vulnerability of ALS: 'diseasedefining' and 'disease-defying' brain regions. Amyotroph. Lateral Scler. Frontotemporal Degener. 17(7-8), 1-10 (2016). 